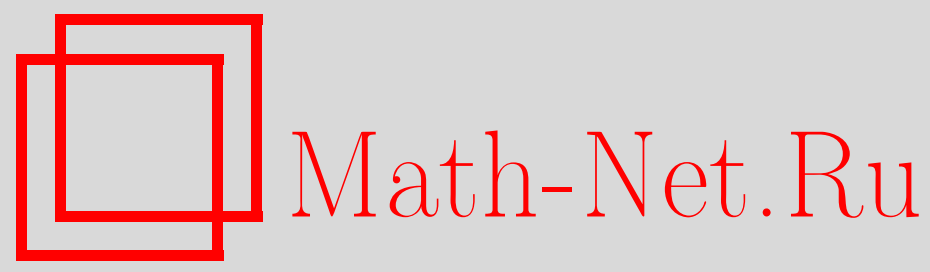

М. Н. Шеремета, О последовательностях максимальных членов и центральных показателей производных рядов Дирихле, Матем. заметки, 1998, том 63, выпуск 3, 457-467

DOI: https://doi.org/10.4213/mzm1303

Использование Общероссийского математического портала Math-Net.Ru подразумевает, что вы прочитали и согласны с пользовательским соглашением http://www.mathnet.ru/rus/agreement

Параметры загрузки:

IP : 34.229 .45 .116

26 апреля 2023 г., $12: 24: 02$

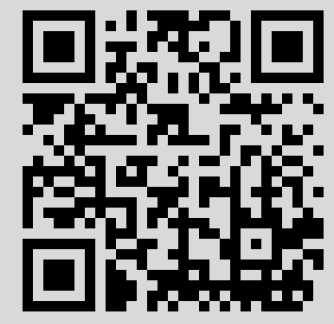




\section{О ПОСЛЕДОВАТЕЛЬНОСТЯХ МАКСИМАЛЬНЫХ ЧЛЕНОВ \\ И ЦЕНТРАЛЬНЫХ ПОКАЗАТЕЛЕЙ ПРОИЗВОДНЫХ РЯДОВ ДИРИХЛЕ}

М.Н. Шеремета

Для представляющего функцию $F$ ряда Дирихле с положительными возрастающими к $\infty$ показателями и абсциссой абсолютной сходимости $A \in(-\infty,+\infty]$ доказано, что последовательности максимальных членов $\left(\mu\left(\sigma, F^{(m)}\right)\right)$ и центральных показателей $\left(\Lambda\left(\sigma, F^{(m)}\right)\right)$ являются неубывающими к $\infty$ при $m \rightarrow \infty$ для любого фиксированного $\sigma<A$, и

$$
\varlimsup_{m \rightarrow \infty} \frac{\ln \mu\left(\sigma, F^{(m)}\right)}{m \ln m} \leqslant 1 \quad \text { и } \quad \varlimsup_{m \rightarrow \infty} \frac{\ln \Lambda\left(\sigma, F^{(m)}\right)}{\ln m} \leqslant 1 .
$$

Указаны необходимые и достаточные условия того, что в этих соотношениях можно поставить знак равенства и заменить

Библиографиял: 3 названия.

1. Введение. Пусть $0<\lambda_{n} \uparrow \infty, n \rightarrow \infty$, а ряд Дирихле

$$
F(s)=\sum_{n=1}^{\infty} a_{n} \exp \left(s \lambda_{n}\right), \quad s=\sigma+i t
$$

имеет абсциссу $A \in(-\infty,+\infty]$ абсолютной сходимости. Будем считать, что все $a_{n} \neq 0$, так что ряд (1) не сводится к экспоненциальному полиному. Для $\sigma \in(-\infty, A)$ пусть $\mu(\sigma, F)=\max \left\{\left|a_{n}\right| \exp \left(\sigma \lambda_{n}\right): n \geqslant 1\right\}-$ максимальный член, $\nu(\sigma, F)=\max \{n \geqslant 1:$ $\left.\left|a_{n}\right| \exp \left(\sigma \lambda_{n}\right)=\mu(\sigma, F)\right\}-$ центральный индекс, а $\Lambda(\sigma, F)=\lambda_{\nu(\sigma, F)}$ - центральный показатель ряда (1).

В настоящей статье исследуется при $m \rightarrow \infty$ асимптотическое поведение для каждого фиксированного $\sigma \in(-\infty, A)$ последовательностей $\left(\mu\left(\sigma, F^{(m)}\right)\right)$ и $\left(\Lambda\left(\sigma, F^{(m)}\right)\right)$, где $F^{(m)}$ - производная функции $F$ порядка $m \in \mathbb{Z}_{+}$. Имеет место следующая

Теорема 1. Для кажсдого фиксированного $\sigma \in(-\infty, A)$ последовательности $\left(\mu\left(\sigma, F^{(m)}\right)\right)$ u $\left(\Lambda\left(\sigma, F^{(m)}\right)\right)$ неубивающие и стремятся $\kappa \infty$ при $m \rightarrow \infty$.

На скорость роста этих последовательностей указывает 
Теорема 2. Для каждого фиксированного $\sigma \in(-\infty, A)$

$$
\varlimsup_{m \rightarrow \infty} \frac{\ln \mu\left(\sigma, F^{(m)}\right)}{m \ln m} \leqslant 1
$$

$u$

$$
\varlimsup_{m \rightarrow \infty} \frac{\ln \Lambda\left(\sigma, F^{(m)}\right)}{\ln m} \leqslant 1 .
$$

Оценки (2) и (3) точны. Это вытекает из следующей теоремы.

Tеорема 3. Пусть $\sigma \in(-\infty, A)$. Для того чтобь

$$
\varlimsup_{m \rightarrow \infty} \frac{\ln \mu\left(\sigma, F^{(m)}\right)}{m \ln m}=1
$$

$u$

$$
\varlimsup_{m \rightarrow \infty} \frac{\ln \Lambda\left(\sigma, F^{(m)}\right)}{\ln m}=1,
$$

необходимо и достаточно, чтобы

$$
\varliminf_{n \rightarrow \infty} \frac{1}{\ln \lambda_{n}} \ln \left(\frac{1}{\lambda_{n}} \ln \frac{1}{\left|a_{n}\right|}-\sigma\right)=0 .
$$

Естественным является вопрос, когда в (4) и (5) можно заменить $\varlimsup$ на $\lim$ ? Ответ на него дает

Teоpema 4. Пусть $\sigma \in(-\infty, A)$. Для того чтобь

$$
\lim _{m \rightarrow \infty} \frac{\ln \mu\left(\sigma, F^{(m)}\right)}{m \ln m}=1
$$

$u$

$$
\lim _{m \rightarrow \infty} \frac{\ln \Lambda\left(\sigma, F^{(m)}\right)}{\ln m}=1,
$$

необходимо и достаточно, чтобы для любого $\varepsilon>0$

1) при $n \geqslant n_{0}(\varepsilon)$ выполнялось неравенство

$$
\frac{1}{\ln \lambda_{n}} \ln \left(\frac{1}{\lambda_{n}} \ln \frac{1}{\left|a_{n}\right|}-\sigma\right)>-\varepsilon ;
$$

2) существовала возрастающая последовательность $\left(n_{k}\right)$ натуральных чисел такая, что $\ln \lambda_{n_{k+1}} \sim \ln \lambda_{n_{k}}$ при $k \rightarrow \infty u$

$$
\frac{1}{\ln \lambda_{n_{k}}} \ln \left(\frac{1}{\lambda_{n_{k}}} \ln \frac{1}{\left|a_{n_{k}}\right|}-\sigma\right)<\varepsilon
$$

Некоторые следствия из теорем 3 и 4 приведены в конце статьи. 
2. Вспомогательные результаты. Пусть $\Omega$-класс положительных неограниченных на $(-\infty,+\infty)$ функций $\Phi$ таких, что производная $\Phi^{\prime}$ непрерывная, положительная и возрастающая к $+\infty$ на $(-\infty,+\infty)$. Обозначим через $\varphi$ функцию, обратную к $\Phi^{\prime}$. Пусть $\Psi(\sigma)=\sigma-\Phi(\sigma) / \Phi^{\prime}(\sigma)$ - функция, ассоциированная с $\Phi$ по Ньютону.

Лемма 1 [1]. Пусть $\Phi \in \Omega$ и ряд Дирихле (1) имеет абсииссу абсолютной сходимости $A=+\infty$. Для того чтобы $\ln \mu(\sigma, F) \leqslant \Phi(\sigma)$ для всех $\sigma \geqslant \sigma_{0}$, необходимо и достаточно, чтобы $\ln \left|a_{n}\right| \leqslant-\lambda_{n} \Psi\left(\varphi\left(\lambda_{n}\right)\right)$ для всех $n \geqslant n_{0}$.

Для произвольного $\varepsilon>0$ функцию $\Phi \in \Omega$ выберем так, чтобы $\Phi(\sigma)=(1+\varepsilon) \sigma \ln \sigma$ для всех достаточно больших $\sigma$. Тогда $\Phi^{\prime}(\sigma)=(1+\varepsilon)(\ln \sigma+1), \Psi(\sigma)=\sigma /(\ln \sigma+1)$, $\varphi(t)=\exp (t /(1+\varepsilon)-1)$ и $t \Psi(\varphi(t))=(1+\varepsilon) \exp (t /(1+\varepsilon)-1)$. Поэтому по лемме 1 для того чтобы $\ln \mu(\sigma, F) \leqslant(1+\varepsilon) \sigma \ln \sigma$ для всех достаточно больших $\sigma$, необходимо и достаточно, чтобы $\ln \left|a_{n}\right| \leqslant-(1+\varepsilon) \exp \left(\lambda_{n} /(1+\varepsilon)-1\right)$ для всех достаточно больших $n$. Отсюда вытекает справедливость следующего утверждения.

ЛЕмма 2. Пусть ряд Дирихле (1) имеет абсциссу абсолютной сходимости $A=$ $+\infty$. Для того чтобь

$$
\varlimsup_{\sigma \rightarrow+\infty} \frac{\ln \mu(\sigma, F)}{\sigma \ln \sigma}=1
$$

необходимо и достаточно, чтобы

$$
\varlimsup_{n \rightarrow \infty} \frac{\lambda_{n}}{\ln \ln \left(1 /\left|a_{n}\right|\right)}=1 .
$$

Следующая лемма интересна сама по себе и указьвает на необходимое и достаточное условие того, что в (11) можно заменить $\varlimsup$ im на $\lim$.

ЛЕмма 3. Пусть ряд Дирихле (1) имеет абсииссу абсолютной сходимости $A=$ $+\infty$. Для того чтобь

$$
\lim _{\sigma \rightarrow+\infty} \frac{\ln \mu(\sigma, F)}{\sigma \ln \sigma}=1,
$$

необходимо и достаточно, чтобы для любого $\varepsilon>0$

1) $п р и ~ n \geqslant n_{0}(\varepsilon)$ выполнялось неравенство

$$
\lambda_{n}<(1+\varepsilon) \ln \ln \frac{1}{\left|a_{n}\right|}
$$

2) существовала возрастающая последовательность $\left(n_{k}\right)$ натуральных чисел такая, что $\lambda_{n_{k+1}} \sim \lambda_{n_{k}}$ npu $k \rightarrow \infty u$

$$
\lambda_{n_{k}}>(1-\varepsilon) \ln \ln \frac{1}{\left|a_{n_{k}}\right|} .
$$


ДоКАЗАТЕЛЬСТво. Начнем с необходимости. Если вьполняется условие (13), то справедливо равенство (12), из которого вытекает свойство 1) леммы 3.

Свойство 2) будем доказывать от противного. Предположим, что существуют числа $\tau>0, \eta \in(0,1)$ и последовательность интервалов $\left(n_{\nu}^{\prime}, n_{\nu}^{\prime \prime}\right), n_{\nu}^{\prime} \rightarrow \infty(\nu \rightarrow \infty)$, такие, что $\lambda_{n_{\nu}^{\prime \prime}}>(1+\tau) \lambda_{n_{\nu}^{\prime}}$ и

$$
\lambda_{n}<(1-\eta) \ln \ln \frac{1}{\left|a_{n}\right|}, \quad n_{\nu}^{\prime}<n<n_{\nu}^{\prime \prime} .
$$

Положим $\sigma_{\nu}=\exp \left((1+\tau / 2) \lambda_{n_{\nu}^{\prime}}\right)$ и рассмотрим ряд Дирихле

$$
F_{*}(s)=\left(\sum_{n \leqslant n_{\nu}^{\prime}}+\sum_{n \geqslant n_{\nu}^{\prime \prime}}\right) b_{n} e^{s \lambda_{n}}, \quad b_{n}=\exp \left(-\exp \left(\frac{\lambda_{n}}{1+\varepsilon}\right)\right),
$$

где $\varepsilon \in(0, \tau /(2+\tau))$. Из (14) вытекает, что $\left|a_{n}\right|<b_{n}$ для $n \leqslant n_{\nu}^{\prime}$ и $n \geqslant n_{\nu}^{\prime \prime}$.

Если $n \leqslant n_{\nu}^{\prime}$, то

$$
\frac{\ln b_{n}-\ln b_{n_{\nu}^{\prime}}}{\lambda_{n_{\nu}^{\prime}}-\lambda_{n}} \leqslant \frac{1}{1+\varepsilon} \exp \left(\frac{\lambda_{n_{\nu}^{\prime}}}{1+\varepsilon}\right) \leqslant \exp \left(\left(1+\frac{\tau}{2}\right) \lambda_{n_{\nu}^{\prime}}\right)=\sigma_{\nu}
$$

а если $n \geqslant n_{\nu}^{\prime \prime}$, то

$$
\begin{aligned}
\frac{\ln b_{n_{\nu}^{\prime \prime}}-\ln b_{n}}{\lambda_{n}-\lambda_{n_{\nu}^{\prime \prime}}} & \geqslant \frac{1}{1+\varepsilon} \exp \left(\frac{\lambda_{n_{\nu}^{\prime \prime}}}{1+\varepsilon}\right) \geqslant \frac{1}{1+\varepsilon} \exp \left(\frac{1+\tau}{1+\varepsilon} \lambda_{n_{\nu}^{\prime}}\right) \\
& =\frac{1}{1+\varepsilon} \exp \left(\frac{1+\tau}{(1+\varepsilon)(1+\tau / 2)} \ln \sigma_{\nu}\right) \geqslant \sigma_{\nu}, \quad \nu \geqslant \nu_{0} .
\end{aligned}
$$

Поэтому для $n \leqslant n_{\nu}^{\prime}$

$$
\ln \left|a_{n}\right|+\sigma_{\nu} \lambda_{n} \leqslant \ln b_{n}+\sigma_{\nu} \lambda_{n} \leqslant \ln b_{n_{\nu}^{\prime}}+\sigma_{\nu} \lambda_{n_{\nu}^{\prime}} \leqslant \sigma_{\nu} \lambda_{n_{\nu}^{\prime}}=\frac{1}{1+\tau / 2} \sigma_{\nu} \ln \sigma_{\nu},
$$

а при $n \geqslant n_{\nu}^{\prime \prime}$

$$
\begin{aligned}
\ln \left|a_{n}\right|+\sigma_{\nu} \lambda_{n} & \leqslant \ln b_{n_{\nu}^{\prime \prime}}+\sigma_{\nu} \lambda_{n_{\nu}^{\prime \prime}}=-\exp \left(\frac{\lambda_{n_{\nu}^{\prime \prime}}}{1+\varepsilon}\right)+\lambda_{n_{\nu}^{\prime \prime}} \exp \left(\left(1+\frac{\tau}{2}\right) \lambda_{n_{\nu}^{\prime}}\right) \\
& \leqslant-\exp \left(\frac{\lambda_{n_{\nu}^{\prime \prime}}}{1+\varepsilon}\right)+\lambda_{n_{\nu}^{\prime \prime}} \exp \left(\frac{1+\tau / 2}{1+\tau} \lambda_{n_{\nu}^{\prime \prime}}\right) \rightarrow-\infty, \quad \nu \rightarrow \infty .
\end{aligned}
$$

Наконец, если $n_{\nu}^{\prime}<n<n_{\nu}^{\prime \prime}$, то из (16) имеем

$$
\begin{aligned}
\ln \left|a_{n}\right|+\sigma_{\nu} \lambda_{n} & \leqslant-\exp \left(\frac{\lambda_{n}}{1-\eta}\right)+\sigma_{\nu} \lambda_{n} \leqslant \max \left\{-\exp \left(\frac{t}{1-\eta}\right)+\sigma_{\nu} t: t \geqslant 0\right\} \\
& =(1+o(1))(1-\eta) \sigma_{\nu} \ln \sigma_{\nu}, \quad \nu \rightarrow \infty .
\end{aligned}
$$


Из (17)-(19) следует, что

$$
\ln \mu\left(\sigma_{\nu}, F\right) \leqslant(1+o(1)) \max \left\{\frac{2}{2+\tau}, 1-\eta\right\} \sigma_{\nu} \ln \sigma_{\nu}, \quad \nu \rightarrow \infty,
$$

а это невозможно в силу (13).

Перейдем к доказательству достаточности. Из условия 1) вытекает, что

$$
\varlimsup_{\sigma \rightarrow+\infty} \frac{\ln \mu(\sigma, F)}{\sigma \ln \sigma} \leqslant 1 .
$$

Далее, рассмотрим ряд Дирихле

$$
F^{*}(s)=\sum_{k=1}^{\infty} b_{k}^{*} \exp \left(s \lambda_{n_{k}}\right), \quad b_{k}^{*}=\exp \left(-\exp \left(\frac{\lambda_{n_{k}}}{1-\varepsilon}\right)\right) .
$$

Из (15) следует, что $\ln \mu(\sigma, F) \geqslant \ln \mu\left(\sigma, F^{*}\right)$. Положим

$$
\varkappa_{k}=\frac{\ln b_{k-1}^{*}-\ln b_{k}^{*}}{\lambda_{n_{k}}-\lambda_{n_{k-1}}} \text {. }
$$

Заметим, что $\varkappa_{k} \uparrow \infty, k \rightarrow \infty$. Нетрудно показать, что

$$
\ln \mu\left(\sigma, F^{*}\right)=\ln b_{k}^{*}+\sigma \lambda_{n_{k}}, \quad \varkappa_{k} \leqslant \sigma \leqslant \varkappa_{k+1} .
$$

$\mathrm{Ha}\left[\varkappa_{k}, \varkappa_{k+1}\right]$ функция $\ln \mu\left(\sigma, F^{*}\right) /(\sigma \ln \sigma)$ вогнутая, поэтому

Далее,

$$
\min \left\{\frac{\ln \mu\left(\sigma, F^{*}\right)}{\sigma \ln \sigma}: \varkappa_{k} \leqslant \sigma \leqslant \varkappa_{k+1}\right\}=\min \left\{\frac{\ln \mu\left(\varkappa_{k}, F^{*}\right)}{\varkappa_{k} \ln \varkappa_{k}}, \frac{\ln \mu\left(\varkappa_{k+1}, F^{*}\right)}{\varkappa_{k+1} \ln \varkappa_{k+1}}\right\} .
$$

$$
\frac{\ln \mu\left(\varkappa_{k}, F^{*}\right)}{\varkappa_{k} \ln \varkappa_{k}}=\frac{\ln b_{k}^{*}+\varkappa_{k} \lambda_{n_{k}}}{\varkappa_{k} \ln \varkappa_{k}}=\frac{\lambda_{n_{k}} \ln b_{k-1}^{*}-\lambda_{n_{k-1}} \ln b_{k}^{*}}{\left(\ln b_{k-1}^{*}-\ln b_{k}^{*}\right) \ln \varkappa_{k}}=\frac{\lambda_{n_{k}} \lambda_{n_{k-1}}}{\ln \varkappa_{k}} \beta_{k},
$$

где

$$
\begin{aligned}
\beta_{k} & =\frac{\left(\ln b_{k-1}^{*}\right) / \lambda_{n_{k-1}}-\left(\ln b_{k}^{*}\right) / \lambda_{n_{k}}}{\ln b_{k-1}^{*}-\ln b_{k}^{*}}=\frac{\exp \left(\frac{\lambda_{n_{k}}}{1-\varepsilon}\right) / \lambda_{n_{k}}-\exp \left(\frac{\lambda_{n_{k-1}}}{1-\varepsilon}\right) / \lambda_{n_{k-1}}}{\exp \left(\frac{\lambda_{n_{k}}}{1-\varepsilon}\right)-\exp \left(\frac{\lambda_{n_{k-1}}}{1-\varepsilon}\right)} \\
& =\frac{1}{1-\varepsilon}\left(\frac{1}{\xi_{k}}-\frac{1}{\xi_{k}^{2}}\right), \quad \frac{\lambda_{n_{k-1}}}{1-\varepsilon}<\xi_{k}<\frac{\lambda_{n_{k}}}{1-\varepsilon},
\end{aligned}
$$

т.е. в силу условия $\lambda_{n_{k}} \sim \lambda_{n_{k-1}}, k \rightarrow \infty$, имеем

$$
\beta_{k}=(1+o(1)) \lambda_{n_{k-1}}, \quad k \rightarrow \infty \text {. }
$$

Аналогично,

т.е.

$$
\ln \varkappa_{k}=\ln \frac{\exp \left(\frac{\lambda_{n_{k}}}{1-\varepsilon}\right)-\exp \left(\frac{\lambda_{n_{k-1}}}{1-\varepsilon}\right)}{\lambda_{n_{k}}-\lambda_{n_{k-1}}}=\ln \left(\frac{1}{1-\varepsilon} e^{\eta_{k}}\right), \quad \frac{\lambda_{n_{k-1}}}{1-\varepsilon}<\eta_{k}<\frac{\lambda_{n_{k}}}{1-\varepsilon},
$$

$$
\ln \varkappa_{k}=\frac{1+o(1)}{1-\varepsilon} \lambda_{n_{k}}, \quad k \rightarrow \infty .
$$

Значит, из (21) следует, что

поэтому

$$
\frac{\ln \mu\left(\varkappa_{k}, F^{*}\right)}{\varkappa_{k} \ln \varkappa_{k}}=(1+o(1))(1-\varepsilon), \quad k \rightarrow \infty ;
$$

$$
\varliminf_{\sigma \rightarrow+\infty} \frac{\ln \mu(\sigma, F)}{\sigma \ln \sigma} \geqslant \varliminf_{\sigma \rightarrow+\infty} \frac{\ln \mu\left(\sigma, F^{*}\right)}{\sigma \ln \sigma} \geqslant 1-\varepsilon .
$$

Из (20) и (22) в силу произвольности $\varepsilon$ получаем (13). Лемма 3 полностью доказана. 
Лемма 4. Пусть ряд Дирихле (1) имеет абсииссу абсолютной сходимости $A=$ $+\infty$. Для того чтобы

$$
\varlimsup_{\sigma \rightarrow+\infty} \frac{\Lambda(\sigma, F)}{\ln \sigma}=1
$$

необходимо и достаточно, чтобы выполнялось равенство (11), а для того чтобы

$$
\lim _{\sigma \rightarrow+\infty} \frac{\Lambda(\sigma, F)}{\ln \sigma}=1
$$

необходимо и достаточно, чтобы выполнялось равенство (13).

ДокАЗАТЕЛЬСтво. Так как

$$
\ln \mu(\sigma, F)=\ln \mu(0, F)+\int_{0}^{\sigma} \Lambda(t, F) d t
$$

по правилу Лопиталя

$$
\varlimsup_{\sigma \rightarrow+\infty} \frac{\ln \mu(\sigma, F)}{\sigma \ln \sigma} \leqslant \varlimsup_{\sigma \rightarrow+\infty} \frac{\Lambda(\sigma, F)}{\ln \sigma} .
$$

С другой стороны, А.В. Братищев [2] показал, что если $f$ и $g$-выпуклые на $[a,+\infty)$ функции, $g^{\prime}(\sigma) \rightarrow+\infty, \sigma \rightarrow+\infty$, и

то

$$
\varlimsup_{\sigma \rightarrow+\infty} \frac{1}{g^{\prime}(\sigma)} \inf _{t>\sigma} \frac{g(t)}{t-\sigma} \leqslant 1
$$

$$
\varlimsup_{\sigma \rightarrow+\infty} \frac{f(\sigma)}{g(\sigma)} \leqslant 1 \Longrightarrow \varlimsup_{\sigma \rightarrow+\infty} \frac{f^{\prime}(\sigma)}{g^{\prime}(\sigma)} \leqslant 1 .
$$

Легко видеть, что функция $g(\sigma)=\sigma \ln \sigma$ удовлетворяет условию (26), так как

$$
g^{\prime}(\sigma)=\ln \sigma+1 \quad \text { и } \quad \inf \left\{\frac{t \ln t}{t-\sigma}: t>\sigma\right\}=\frac{t(\sigma) \ln t(\sigma)}{t(\sigma)-\sigma},
$$

где $t(\sigma)=\sigma \ln \sigma+\sigma \ln \ln \sigma+\sigma+o(1), \sigma \rightarrow+\infty$, и значит,

$$
\inf \left\{\frac{t \ln t}{t-\sigma}: t>\sigma\right\}=(1+o(1)) \frac{\sigma \ln ^{2} \sigma}{\sigma \ln \sigma}=(1+o(1)) \ln \sigma, \quad \sigma \rightarrow+\infty .
$$

Поэтому

$$
\varlimsup_{\sigma \rightarrow+\infty} \frac{\ln \mu(\sigma, F)}{\sigma \ln \sigma} \leqslant 1 \Longrightarrow \varlimsup_{\sigma \rightarrow+\infty} \frac{\Lambda(\sigma, F)}{\ln \sigma} \leqslant 1 .
$$

Из (25) и (27) получаем эквивалентность равенств (11) и (23).

В [2] также показано, что если $g^{\prime}(\sigma) \uparrow+\infty, \sigma \rightarrow+\infty$, и для каждого $\varepsilon \in(0,1)$

$$
\alpha(\varepsilon)=\varlimsup_{\sigma \rightarrow+\infty} \frac{g\left(\sigma^{*}\right)+g^{\prime}\left(\sigma^{*}\right)\left(\sigma-\sigma^{*}\right)}{g(\sigma)}<1,
$$

где $g^{\prime}\left(\sigma^{*}\right)=(1-\varepsilon) g^{\prime}(\sigma)$, то

$$
\lim _{\sigma \rightarrow+\infty} \frac{f(\sigma)}{g(\sigma)}=1 \Longrightarrow \lim _{\sigma \rightarrow+\infty} \frac{f^{\prime}(\sigma)}{g^{\prime}(\sigma)}=1 .
$$

В нашем случае $g(\sigma)=\sigma \ln \sigma, \sigma^{*}=\exp ((1-\varepsilon) \ln \sigma-\varepsilon)$ и $\alpha(\varepsilon)=1-\varepsilon$. Поэтому

$$
\lim _{\sigma \rightarrow+\infty} \frac{\ln \mu(\sigma, F)}{\sigma \ln \sigma}=1 \Longrightarrow \lim _{\sigma \rightarrow+\infty} \frac{\Lambda(\sigma, F)}{\ln \sigma}=1,
$$

что вместе с правилом Лопиталя указьвает на эквивалентность равенств (13) и (24). 
3. Доказательство теоремы 1. Покажем сначала, что

$$
\Lambda\left(\sigma, F^{(m)}\right) \leqslant \frac{\mu\left(\sigma, F^{(m+1)}\right)}{\mu\left(\sigma, F^{(m)}\right)} \leqslant \Lambda\left(\sigma, F^{(m+1)}\right), \quad m \in \mathbb{Z}_{+}, \quad \sigma \in(-\infty, A) .
$$

Действительно, с одной стороны,

$$
\begin{aligned}
\mu\left(\sigma, F^{(m)}\right) & =\left|a_{\nu\left(\sigma, F^{(m)}\right)}\right| \lambda_{\nu\left(\sigma, F^{(m)}\right)}^{m} \exp \left(\sigma \lambda_{\nu\left(\sigma, F^{(m)}\right)}\right) \\
& =\frac{1}{\Lambda\left(\sigma, F^{(m)}\right)}\left|a_{\nu\left(\sigma, F^{(m)}\right)}\right| \lambda_{\nu\left(\sigma, F^{(m)}\right)}^{m+1} \exp \left(\sigma \lambda_{\nu\left(\sigma, F^{(m)}\right)}\right) \leqslant \frac{\mu\left(\sigma, F^{(m+1)}\right)}{\Lambda\left(\sigma, F^{(m)}\right)}
\end{aligned}
$$

т.е. выполнено левое неравенство в (28). С другой стороны,

$$
\begin{aligned}
\mu\left(\sigma, F^{(m+1)}\right) & =\left|a_{\nu\left(\sigma, F^{(m+1)}\right)}\right| \lambda_{\nu\left(\sigma, F^{(m+1)}\right)}^{m+1} \exp \left(\sigma \lambda_{\nu\left(\sigma, F^{(m+1)}\right)}\right) \\
& =\Lambda\left(\sigma, F^{(m+1)}\right)\left|a_{\nu\left(\sigma, F^{(m+1)}\right)}\right| \lambda_{\nu\left(\sigma, F^{(m+1)}\right)}^{m} \exp \left(\sigma \lambda_{\nu\left(\sigma, F^{(m+1)}\right)}\right) \\
& \leqslant \Lambda\left(\sigma, F^{(m+1)}\right) \mu\left(\sigma, F^{(m)}\right),
\end{aligned}
$$

т.е. получаем правое неравенство в (28).

Из $(28)$ следует, что последовательности $\left(\Lambda\left(\sigma, F^{(m)}\right)\right)$ и $\left(\mu\left(\sigma, F^{(m)}\right)\right)$ не убьвают. Так как $\lambda_{n} \uparrow+\infty$, то и последовательность $\left(\nu\left(\sigma, F^{(m)}\right)\right)$ также неубывающая, и чтобы доказать стремление $\Lambda\left(\sigma, F^{(m)}\right)$ к $\infty$ при $m \rightarrow \infty$, достаточно показать, что таким свойством обладает последовательность $\left(\nu\left(\sigma, F^{(m)}\right)\right)$.

Предположим противное: последовательность $\left(\nu\left(\sigma, F^{(m)}\right)\right)$ ограничена. Так как $\nu(\sigma$, $\left.F^{(m)}\right)$ - целочисленная функция, существует $p=p(\sigma) \in \mathbb{N}$ такое, что $\nu\left(\sigma, F^{(m)}\right)=p$ для всех $m \geqslant p$. Но все $a_{n} \neq 0$. Поэтому

$1>\frac{\left|a_{p+1}\right| \lambda_{p+1}^{m} \exp \left(\sigma \lambda_{p+1}\right)}{\left|a_{p}\right| \lambda_{p}^{m} \exp \left(\sigma \lambda_{p}\right)}=\left|\frac{a_{p+1}}{a_{p}}\right| \exp \left(\sigma\left(\lambda_{p+1}-\lambda_{p}\right)\right)\left(\frac{\lambda_{p+1}}{\lambda_{p}}\right)^{m} \rightarrow+\infty, \quad m \rightarrow \infty$

что невозможно. Значит, $\Lambda\left(\sigma, F^{(m)}\right) \rightarrow \infty, m \rightarrow \infty$, и в силу $(28) \mu\left(\sigma, F^{(m)}\right) \rightarrow \infty$, $m \rightarrow \infty$.

4. Доказательство теоремы 2. Сначала заметим следующее. Предположим, что функциональная последовательность $F(\sigma)=\left(\left|a_{n}\right| \exp \left(\sigma \lambda_{n}\right)\right)_{n=1}^{\infty}$ удовлетворяет условию $\left|a_{n}\right| \exp \left(\sigma \lambda_{n}\right) \rightarrow 0, n \rightarrow \infty$, для любого $\sigma \in \mathbb{R}$. Тогда для любого $\sigma \in \mathbb{R}$ существует максимальньй член $\mu(\sigma, F)$ этой последовательности и все утверждения, полученные для максимального члена абсолютно сходящегося в $\mathbb{C}$ ряда Дирихле $(1)$, остаются верными и для максимального члена указанной функциональной последовательности. В частности, справедливы все леммы, приведенные в п. 2.

Не уменьшая общности, будем считать, что $\lambda_{1} \geqslant 1$, и для каждого фиксированного $\sigma \in(-\infty, A)$, где $A \in(-\infty,+\infty]$ - абсцисса абсолютной сходимости ряда $(1)$, рассмотрим функциональную последовательность

$$
F_{\sigma}(x)=\left(\left|a_{n}\right| e^{\sigma \lambda_{n}} e^{x \ln \lambda_{n}}\right)_{n=1}^{\infty}, \quad x \in \mathbb{R}
$$


Покажем, что общий член этой последовательности для любого $x \in \mathbb{R}$ стремится к 0 при $n \rightarrow \infty$. Для этого, прежде всего, заметим, что

$$
\varlimsup_{n \rightarrow \infty} \frac{1}{\lambda_{n}} \ln \left|a_{n}\right| \leqslant-A .
$$

Действительно, если $A<+\infty$ и сушествуют число $\eta>0$ и возрастающая последовательность натуральных чисел такие, что

$$
\frac{1}{\lambda_{n_{k}}} \ln \left|a_{n_{k}}\right|>-A+\eta
$$

то для $\sigma \in[A-\eta, A)$ имеем

$$
\left|a_{n_{k}}\right| \exp \left(\sigma \lambda_{n_{k}}\right) \geqslant \exp \left((\sigma-A+\eta) \lambda_{n_{k}}\right) \geqslant 1,
$$

что невозможно, так как $A$ - абсцисса абсолютной сходимости ряда (1). В случае $A=+\infty$ доказательство (30) аналогично.

Из неравенства $(30)$ в случае $A \in(-\infty,+\infty)$ при $n \geqslant n_{0}(\sigma)$ имеем

$$
\begin{aligned}
\left|a_{n}\right| e^{\sigma \lambda_{n}} e^{x \ln \lambda_{n}} & =\exp \left(\lambda_{n}\left(\frac{\ln \left|a_{n}\right|}{\lambda_{n}}+\sigma\right)+x \ln \lambda_{n}\right) \\
& \leqslant \exp \left(\lambda_{n}\left(\sigma-A+\frac{A-\sigma}{2}\right)+x \ln \lambda_{n}\right) \leqslant \exp \left(-\frac{A-\sigma}{2} \lambda_{n}+x \ln \lambda_{n}\right),
\end{aligned}
$$

а в случае $A=+\infty$ вьполняется

$$
\left|a_{n}\right| e^{\sigma \lambda_{n}} e^{x \ln \lambda_{n}}=\exp \left(-\lambda_{n}\left(\frac{1}{\lambda_{n}} \ln \frac{1}{\left|a_{n}\right|}-\sigma\right)+x \ln \lambda_{n}\right) \leqslant \exp \left(-\lambda_{n}+x \ln \lambda_{n}\right),
$$

так как в этом случае, как видно из (30),

$$
\frac{1}{\lambda_{n}} \ln \frac{1}{\left|a_{n}\right|} \rightarrow+\infty, \quad n \rightarrow \infty
$$

Из (31) и $(32)$ вытекает, что максимальньй член $\mu\left(x, F_{\sigma}\right)$ и центральный индекс $\nu\left(x, F_{\sigma}\right)$ последовательности (29) определены для любого $x \in \mathbb{R}$. Из этих неравенств вытекает также, что с определенной постоянной $K=K(\sigma)$ верны соотношения

$\ln \mu\left(x, F_{\sigma}\right) \leqslant \max \{-K t+x \ln t: t \geqslant 1\}=-x+x \ln \frac{x}{K}=(1+o(1)) x \ln x, \quad x \rightarrow+\infty$, т.е.

$$
\varlimsup_{x \rightarrow+\infty} \frac{\ln \mu\left(x, F_{\sigma}\right)}{x \ln x} \leqslant 1 .
$$

Учитьвая то, что у функциональной последовательности (29) показателями являются $\ln \lambda_{n}$, из (34) и (27) имеем

$$
\varlimsup_{x \rightarrow+\infty} \frac{\ln \lambda_{\nu\left(x, F_{\sigma}\right)}}{\ln x} \leqslant 1 .
$$

Для ряда (1)

$$
F^{(m)}(s)=\sum_{n=1}^{\infty} a_{n} \lambda_{n}^{m} e^{s \lambda_{n}}
$$

Поэтому

$$
\mu\left(\sigma, F^{(m)}\right)=\mu\left(m, F_{\sigma}\right), \quad \Lambda\left(\sigma, F^{(m)}\right)=\lambda_{\nu\left(m, F_{\sigma}\right)} .
$$

Значит, из (34) и (35) вытекают неравенства (2) и (3). 
5. Доказательство теорем 3 и 4. Применим лемму 2 к максимальному члену функциональной последовательности (29) и получим, что условие

$$
\varlimsup_{n \rightarrow \infty} \frac{\ln \lambda_{n}}{\ln \ln \left(1 /\left(\left|a_{n}\right| \exp \left(\sigma \lambda_{n}\right)\right)\right)}=1
$$

является необходимым и достаточным, для того чтобы

$$
\varlimsup_{x \rightarrow+\infty} \frac{\ln \mu\left(x, F_{\sigma}\right)}{x \ln x}=1,
$$

а согласно лемме 4 и для того чтобы

$$
\varlimsup_{x \rightarrow+\infty} \frac{\ln \lambda_{\nu\left(x, F_{\sigma}\right)}}{\ln x}=1 .
$$

Нетрудно проверить, что условие (37) равносильно условию (6). В силу (36), неубывания функций $\ln \mu\left(x, F_{\sigma}\right)$ и $\ln \lambda_{\nu\left(x, F_{\sigma}\right)}$ и соотношения

$$
(x+O(1)) \ln (x+O(1))=(1+o(1)) x \ln x, \quad x \rightarrow+\infty,
$$

равенства (38) и (39) эквивалентны соответственно равенствам (4) и (5). Теорема 3 доказана.

Применяя лемму 3 к $\mu\left(x, F_{\sigma}\right)$, получим, что выполнение для любого $\varepsilon>0$ следующих двух условий:

$\left.1^{\prime}\right)$ для всех $n \geqslant n_{0}(\varepsilon)$

$$
\ln \lambda_{n}<(1+\varepsilon) \ln \ln \frac{1}{\left|a_{n}\right| \exp \left(\sigma \lambda_{n}\right)} ;
$$

$\left.2^{\prime}\right)$ существует возрастающая последовательность $\left(n_{k}\right)$ натуральных чисел такая, что $\ln \lambda_{n_{k+1}} \sim \ln \lambda_{n_{k}}, k \rightarrow \infty$ и

$$
\ln \lambda_{n_{k}}>(1-\varepsilon) \ln \ln \frac{1}{\left|a_{n_{k}}\right| \exp \left(\sigma \lambda_{n_{k}}\right)} ;
$$

является необходимым и достаточным, для того чтобы

$$
\lim _{x \rightarrow+\infty} \frac{\ln \mu\left(x, F_{\sigma}\right)}{x \ln x}=1 .
$$

Условия $1^{\prime}$ ) и $2^{\prime}$ ) по лемме 4 также являются необходимыми и достаточными, для того чтобы

$$
\lim _{x \rightarrow+\infty} \frac{\ln \lambda_{\nu\left(x, F_{\sigma}\right)}}{\ln x}=1 .
$$

Нетрудно показать, что неравенство (40) равносильно неравенству (9), а неравенство (41) - неравенству (10). Поэтому условия 1'), $2^{\prime}$ ) эквивалентны условиям 1), 2) теоремы 4. Используя приемы, примененные при доказательстве теоремы 3 , легко показать, что равенство (42) равносильно равенству (7), а равенство (43) - равенству (8). Теорема 4 также доказана. 
6. Приложения. В случае, когда $A=+\infty$, в силу (33) в соотношениях (6), (9) и (10) можно $\sigma$ устранить. Поэтому из теорем 3 и 4 вытекают соответственно следующие утверждения.

СлеДСТвИЕ 1. Пусть $A=+\infty$. Для того чтобы для каждого фиксированного $\sigma \in \mathbb{R}$ выполнялись равенства (4) и (5), необходимо и достаточно, чтобь

$$
\varliminf_{n \rightarrow \infty} \frac{1}{\ln \lambda_{n}} \ln \ln \frac{1}{\left|a_{n}\right|}=1 \text {. }
$$

СлЕДСТВИЕ 2. Пусть $A=+\infty$. Для того чтобы для каждого фиксированного $\sigma \in \mathbb{R}$ выполнялись равенства (7) и (8), необходимо и достаточно, чтобь для любого $\varepsilon>0$

1) $n p u n \geqslant n_{0}(\varepsilon)$

$$
\frac{1}{\ln \lambda_{n}} \ln \ln \frac{1}{\left|a_{n}\right|}>1-\varepsilon ;
$$

2) существовала возрастающая последовательность $\left(n_{k}\right)$ натуральных чисел такая, чmo $\ln \lambda_{n_{k+1}} \sim \ln \lambda_{n_{k}}, k \rightarrow \infty, u$

$$
\frac{1}{\ln \lambda_{n_{k}}} \ln \ln \frac{1}{\left|a_{n_{k}}\right|}<1+\varepsilon .
$$

Случай $A=0$ более богатьй, чем случай $A=+\infty$. Во-первых, существуют ряды Дирихле (1), для которых $A=0$ и вьполнятся (30). Для них остаются в силе утверждения следствий 1 и 2 при всех $\sigma<0$. Примером такого ряда может служить

$$
F(s)=\sum_{n=3}^{\infty} \frac{1}{n \ln n} \exp \left(s \frac{\ln n}{\ln \ln n}\right) .
$$

Во-вторых, если $A=0$ и $\ln n=o\left(\lambda_{n}\right), n \rightarrow \infty$, то по теореме Валирона

$$
\varlimsup_{n \rightarrow \infty} \frac{1}{\lambda_{n}} \ln \left|a_{n}\right|=0
$$

Поэтому (6) выполняется для любого $\sigma<0$, и из теоремы 3 вытекает

СлЕДСТвИЕ 3. Пусть $A=0 u \ln n=o\left(\lambda_{n}\right), n \rightarrow \infty$. Тогда для кажждого фиксированного $\sigma \in(-\infty, 0)$ выполняются равенства (4) $и$ (5).

Из теоремы 4 легко получить

СлЕДСТВИЕ 4. Пусть $A=0, \ln \lambda_{n+1} \sim \ln \lambda_{n}, n \rightarrow \infty, u$

$$
\lim _{n \rightarrow \infty} \frac{1}{\lambda_{n}} \ln \left|a_{n}\right|=0
$$

Тогда выполняются равенства (9) и (10).

Для ряда (1) и $\sigma \in(-\infty, A)$ положим $M(\sigma, F)=\sup \{|F(\sigma+i t)|: t \in \mathbb{R}\}$. Предположим, что $A=+\infty$ и $\ln \left|a_{n}\right| \leqslant-\lambda_{n} \alpha\left(\lambda_{n}\right), n \geqslant n_{0}$, где $\alpha-$ положительная непрерьвная 
возрастающая к $+\infty$ на $[0,+\infty)$ функция. Тогда для того чтобы $\ln M(\sigma, F) \sim \ln \mu(\sigma, F)$ при $\sigma \rightarrow+\infty$, необходимо и достаточно, чтобы $\ln n=O\left(\alpha\left(\lambda_{n}\right)\right), n \rightarrow \infty$ (см. [3]). Если $\ln \mu(\sigma, F) \leqslant(1+o(1)) \sigma \ln \sigma, \sigma \rightarrow+\infty$, то, как при доказательстве леммы 2, имеем

$$
\ln \left|a_{n}\right| \leqslant-\exp \left((1+o(1)) \lambda_{n}\right)=-\lambda_{n} \exp \left((1+o(1)) \lambda_{n}\right), \quad n \rightarrow \infty
$$

Таким образом, $\ln M(\sigma, F) \sim \ln \mu(\sigma, F), \sigma \rightarrow+\infty$, если только

$$
\varlimsup_{n \rightarrow \infty} \frac{\ln \ln n}{\lambda_{n}} \leqslant 1 .
$$

Отсюда следует, что если

$$
\varlimsup_{n \rightarrow \infty} \frac{\ln \ln n}{\ln \lambda_{n}} \leqslant 1
$$

и

$$
F_{\sigma}(x)=\sum_{n=1}^{\infty}\left|a_{n}\right| e^{\sigma \lambda_{n}} e^{x \ln \lambda_{n}}, \quad \sigma<A, \quad x \in \mathbb{R},
$$

то $\ln F_{\sigma}(x) \sim \ln \mu\left(x, F_{\sigma}\right)$ при $x \rightarrow+\infty$. С другой стороны,

$$
\mu\left(m, F_{\sigma}\right)=\mu\left(\sigma, F^{(m)}\right) \leqslant M\left(\sigma, F^{(m)}\right) \leqslant F_{\sigma}(m) .
$$

Поэтому в теоремах 2,3 и следствиях $1-4$ в случае выполнения условия (44) можно заменить $\ln \mu\left(\sigma, F^{(m)}\right)$ на $\ln M\left(\sigma, F^{(m)}\right)$.

\section{СПИСОК ЦИТИРОВАННОЙ ЛИТЕРАТУРЫ}

[1] Шеремета М.Н. Двучленная асимптотика целых рядов Дирихле // Теор. функций, функцион. анализ и их прилож. 1990. Т. 54. С. 16-25.

[2] Братищев А.В. Об обращении правила Лопиталя // Механика сплошной среды. Ростов-на-Дону: Изд-во РГУ, 1985. С. 28-42.

[3] Шеремета М.Н. О соотношениях между максимальным членом и максимумом модуля целого ряда Дирихле // Матем. заметки. 1992. Т. 51. № 5. С. 141-148.

Львовский государственный университет

Поступило

01.04 .96 